\title{
Thermodynamics of the one-dimensional half-filled-band Falicov-Kimball model
}

\author{
C. A. Macêdo, L. G. Azevedo, and A. M. C. de Souza \\ Departamento de Física, Universidade Federal de Sergipe, 49100-000 São Cristóvão, Sergipe, Brazil
}

(Received 6 March 2001; published 25 October 2001)

\begin{abstract}
In order to contribute to understand well the finite-temperature properties of the Falicov-Kimball model, we study the temperature dependence of the specific heat, internal energy, entropy, and mean square fluctuation of the difference between the ion and electron numbers, and of the correlation functions of the one-dimensional half-filled-band Falicov-Kimball model. For this purpose we used the method of small-cluster exactdiagonalization calculations with the application of the grand canonical ensemble and of extrapolation techniques to the infinite chain. Our results show that the examined Falicov-Kimball model exhibits behavior of alternate electron-ion short-range ordering at low temperature, and gradual metal-insulator transition at high temperature, but, does not exhibit the crossing point of the specific heat curves, characteristic of the Hubbard model.
\end{abstract}

DOI: 10.1103/PhysRevB.64.184441

PACS number(s): 75.10.Lp, 71.27.+a, 75.40.Mg, 71.10.Fd

\section{INTRODUCTION}

The Falicov-Kimball model was, originally, created for a semiconductor-metal transition based on the existence of both localized and itinerant interacting quasiparticle states. ${ }^{1}$ It was applied, in this context, to the study of many substances, including $\mathrm{SmB}_{6}, \mathrm{Ti}_{2} \mathrm{O}_{3}, \mathrm{~V}_{2} \mathrm{O}_{3}, \mathrm{VO}, \mathrm{Fe}_{3} \mathrm{O}_{4}$, and $\mathrm{VO}_{2}{ }^{1}$ The Falicov-Kimball model, presently, is the simplest model to study metal-insulator transitions in mixed valence compounds of rare earth and transition metal oxides, ordering in mixed valence systems, order-disorder transitions in binary alloys, itinerant magnetism, and crystallization. ${ }^{1-21}$ Recently, it was also applied to study the possibility of electronic ferroelectricity in mixed-valence compounds, ${ }^{22-24}$ and also of the phase diagram of metal ammonia solutions. ${ }^{25}$ In its most simplified version, namely, static model, it consists in assuming that in the system exist two species of spinless fermions: one of them possess infinite mass and hence does not move while the other one is free to move. This version, in its one-dimensional form for a lattice of $N$ sites, can be written as

$$
H=-t \sum_{i}\left(d_{i}^{\dagger} d_{i+1}+\text { H.c. }\right)+U \sum_{i} d_{i}^{\dagger} d_{i} f_{i}^{\dagger} f_{i},
$$

where $d_{i}^{\dagger}\left(d_{i}\right)$ and $f_{i}^{\dagger}\left(f_{i}\right)$ are, respectively, the creation (annihilation) operators for the itinerant and localized fermions at site $i$ (hereafter, the former are called electrons and the latter ions); $U$ is the Coulombian repulsion that operates when the two fermions occupy the same site; and $t$ is the electron transfer integral connecting states localized on nearestneighbor sites. The number of electrons (ions) on site $i$ is denoted as $n_{d i}=d_{i}^{\dagger} d_{i}\left(n_{f i}=f_{i}^{\dagger} f_{i}\right)$.

In despite of the existence of an impressive research activity since its creation, the properties of the Falicov-Kimball model are far from being understood. ${ }^{26}$ In particular, there exist only a few exact results at finite temperatures of this seemingly simple model. Kennedy and Lieb, ${ }^{6}$ and Brand and Schmidt ${ }^{7}$ have proven that at low temperatures, in systems with bipartite lattices at the symmetry point (half-filled band) the ions tend to arrange themselves on one sublattice, there- fore forming a crystal. Freericks et al. ${ }^{27-29}$, and Brand and Mielsch ${ }^{8}$ studied the limit of large spatial dimensions. Farkašovský ${ }^{30}$ determined the ion-state occupation and the specific heat as functions of temperature and ion-level energy $\left(E_{f}\right)$ for the one-dimensional Falicov-Kimball model using small cluster exact-diagonalization calculations.

In this paper we study the thermodynamic properties of the one-dimensional half-filled-band Falicov-Kimball model using the method of small-cluster exact-diagonalization calculations with the application of the grand canonical ensemble and of extrapolation techniques to the infinite chain. ${ }^{31,32}$ We perform an exhaustive study of the temperature dependence of the specific heat, internal energy, entropy, mean square fluctuation of the difference between the ion and electron numbers, and of the correlation functions for a wide range of values of the relative magnitude of the Coulombian repulsion to transfer integral $(U / t)$. We take the ion-level energy as being null $\left(E_{f}=0\right)$, in the line of Lieb $^{6}$ and de Vries,${ }^{10}$ thus assuming the so-called symmetrical case which measures the energies of electrons and ions from of the same origin. Dealing with the minimum number of parameters ( $t$ and $U$ only) we can establish the thermodynamic properties of the model without the extra effect of a different "effective " chemical potential for ions $\left(\mu_{\text {eff }}=U / 2+E_{f}\right)$ and for electrons $(\mu=U / 2)$ and make possible a matching with the known results of the simple Hubbard model. ${ }^{31,33}$

We compare our results with those available in the literature, obtained with the same method used in our work, for the Hubbard model, thus illustrating the effect of the immobility of ions. We find that the Falicov-Kimball model exhibits characteristic behavior of alternate electron-ion shortrange ordering at low-temperature, and gradual metalinsulator transition at high temperature. These properties are similar to those of the Hubbard model, being that the alternate electron-ion short-range ordering, that in the case of the Hubbard model corresponds to antiferromagnetic short-range ordering, occurs at lower temperatures. Also, the FalicovKimball model does not exhibit the crossing point of the specific heat curves ${ }^{34}$ characteristic of Hubbard model, and we identify similarities between the forms of the specific 


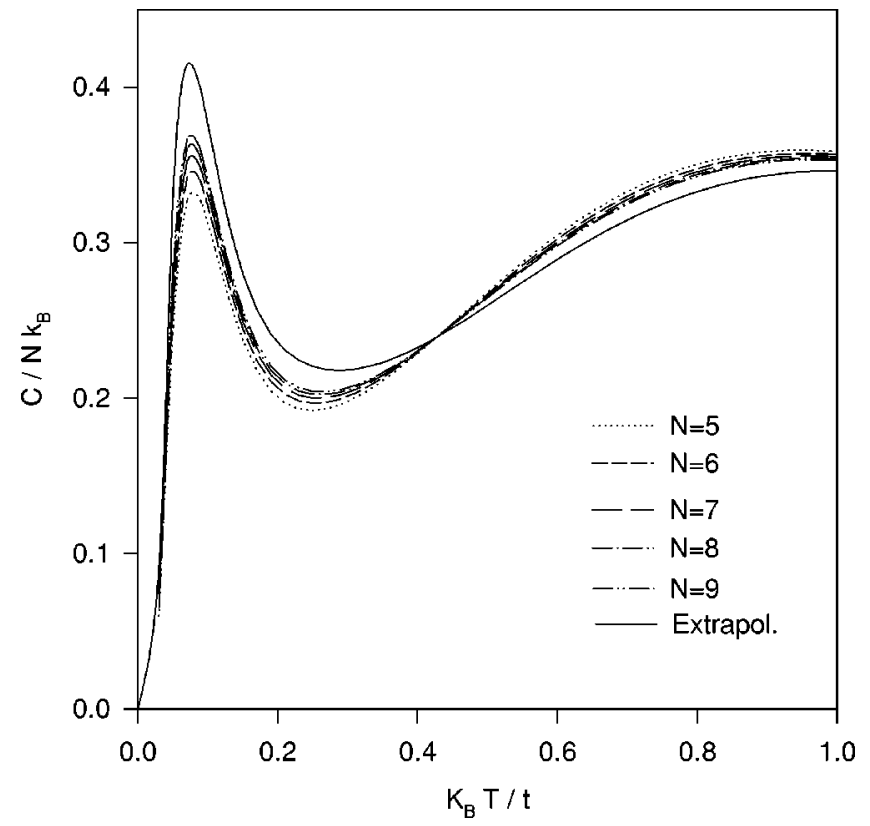

FIG. 1. Specific heat $C / N k_{B}$ of chains with 7 to 9 atoms and rings with 6 and 8 atoms vs temperature for $U / t=4$. The full line is an extrapolation to the infinite chain.

heat curves in low temperatures of the Falicov-Kimball model and of the experimental data of samarium hexaborite. $^{35}$

This paper is organized as follows. In Sec. II we describe our method of computation. In Sec. III the quantities that we calculate are defined, and the results are presented. We close with a conclusion in Sec. IV.

\section{NUMERICAL COMPUTATIONS}

Our approach is to perform exact calculations of thermodynamic quantities in finite one-dimensional half-filled-band systems described by the Hamiltonian (1), with extrapolation to the infinite chain. In the diagonalization of the Hamiltonian, two types of boundary conditions are imposed: chain - a system with free ends or ring - a system with cyclic boundary conditions. The total number of eigenvalues we need is $4^{N}$. The size of the eigenvalue matrix to be diagonalized can be reduced to a large extent by using the symmetry of the system: (i) there is the particle-hole symmetry in rings with even number of sites and in chains; (ii) the total number of each kind of fermion is conserved; (iii) the geometry of the system further simplifies the problem.

Actually we have calculated all eigenvalues and eigenfunctions for chains with 2 to 10 atoms and rings with 6 and 8 atoms. Thermodynamic properties have been evaluated by employing statistical mechanics of the grand canonical ensemble, considering that in the half-filled band with particlehole symmetry the chemical potential $\mu$ is equal to $U / 2$ independently of the temperature. ${ }^{6}$

The extrapolation procedure to the infinite chain is made from a careful analysis of each value of each thermodynamic quantity at each temperature for increasing values of $N$. Also, we tested the consistence of the calculations using the spe-

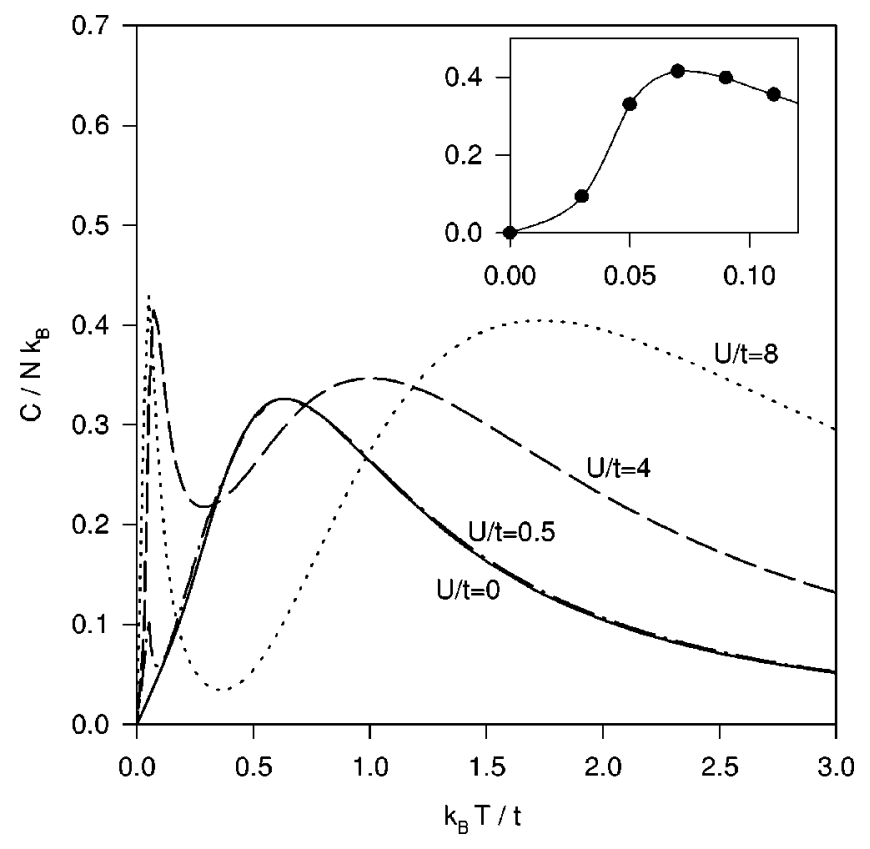

FIG. 2. Specific heat $C / N k_{B}$ of the infinite chain vs. temperature for four typical values of $U / t(0,0.5,4$, and 8$)$. The insert shows details of the specific heat curve for $U / t=4$ in low temperatures.

cific heat and internal energy for $U / t=0$ obtained by direct integration using state density. As an example of the extrapolation technique employed, we show in Fig. 1 the case of the temperature dependence of the specific heat with $U / t=4$.

\section{THERMODYNAMIC PROPERTIES}

In order to present some general features of the FalicovKimball model at finite temperatures we consider several values of $U$ for all the studied thermodynamic properties. We determine with considerable accuracy the temperature dependence of the specific heat, internal energy, entropy, mean square fluctuation of the difference between the ion and electron numbers, and correlation functions of the infinite halffilled-band chain.

\section{A. Specific heat}

The temperature dependence of the specific heat of the infinite chain is shown in Fig. 2 for four typical values of $U / t$. For $U / t=0$ it has a peak at $k_{B} T / t \sim 0.63$. For $U / t>0$ the peak splits in two, which reflects a rearrangement of the fermionic structure in the system. The low-temperature peak arises due to low-lying collective excitations, while the hightemperature broad peak comes from single-particle excitations (or charge-transfer excitations). This conclusion is supported by the behavior of the correlation functions. The characteristic temperature $T_{-}$(defined for $\partial^{2} L_{\delta} /\left.\partial T^{2}\right|_{T=T_{-}}$ $=0, \delta>0$ ) of the decrease of the correlation between the differences of the number of ions to the number of electrons at different sites correspond approximately to the temperature of low-temperature peak. While in the temperature of the high-temperature peak the correlation functions $\left(L_{\delta}, \delta\right.$ $>0)$ are negligible, and the characteristic temperature $T_{+}$ 


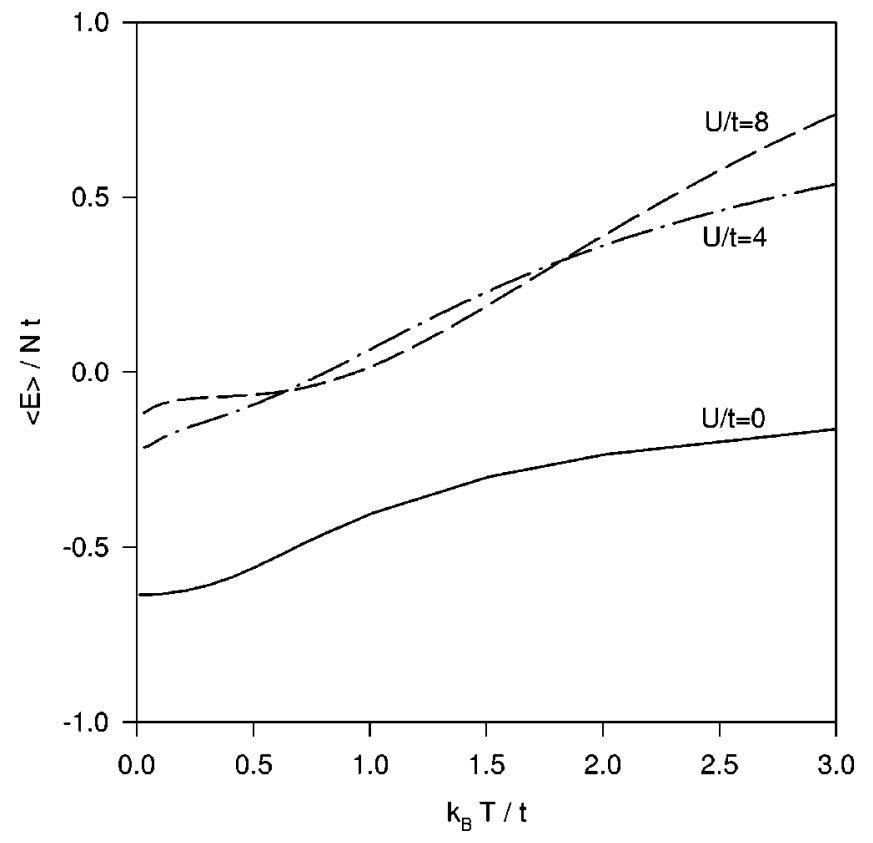

FIG. 3. Internal energy $E / N t$ of the infinite chain vs temperature for $U / t=0,4$, and 8 .

(defined for $\partial^{2} L_{0} /\left.\partial T^{2}\right|_{T=T_{+}}=0$ ) of the decrease of the correlation between the differences of the number of ions to the number of electrons at each site correspond approximately to the temperature of the high-temperature peak. The specific heat $(C)$ of the chain is proportional to $k_{B} T$ at very low temperatures and the coefficient becomes large with $U / t$. The picture presented is analogous to that of the onedimensional half-filled-band Hubbard model, ${ }^{31}$ but the critical value of $U / t$ that causes the splitting of the peaks is drastically different: $U / t \sim 4$ for the Hubbard model and

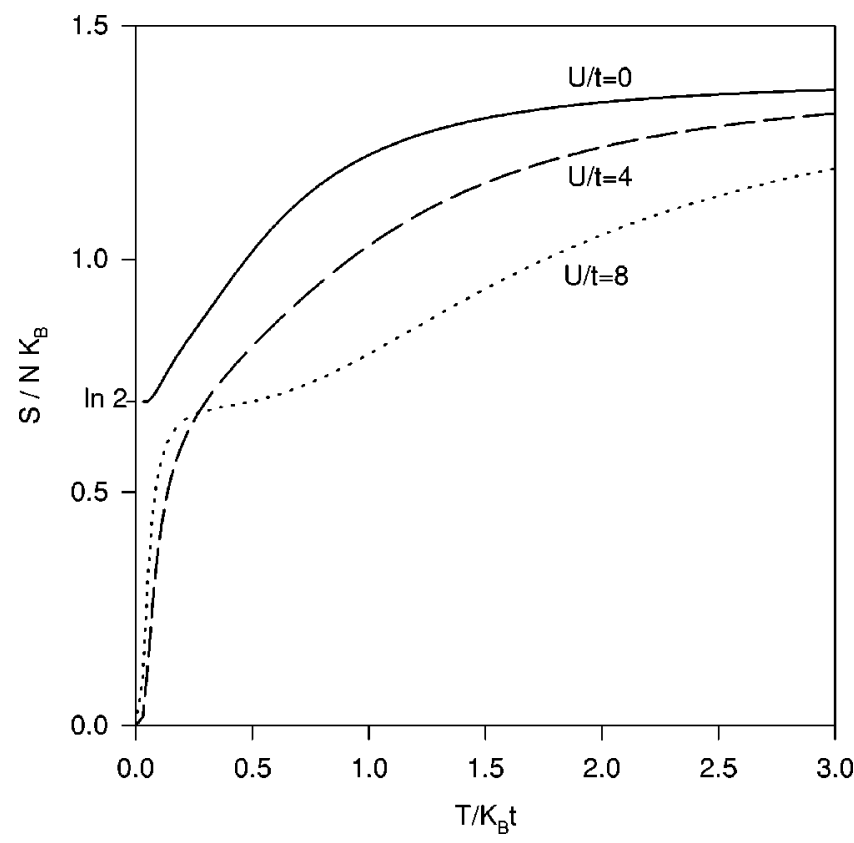

FIG. 4. Entropy $S / N k_{B}$ of the infinite chain vs temperature for $U / t=0,4$, and 8 .

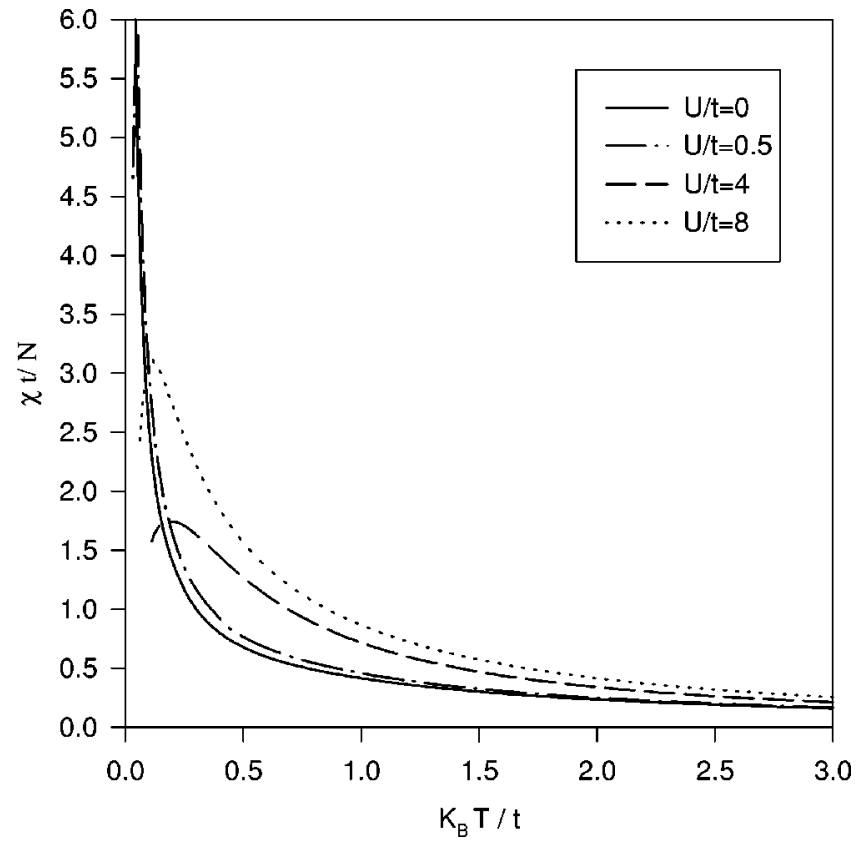

FIG. 5. Mean square fluctuation of the difference between the ion and electron numbers $\chi t / N \mu_{B}^{2}$ of the infinite chain vs temperature for $U / t=0,0.5,4$, and 8 .

$U / t=0$ for the Falicov-Kimball model. This result expresses the importance of the ionic immobility in the case of the Falicov-Kimball model, which makes easy the rearrangement of the fermionic structure in the system as $U / t$ increases. In the one-dimensional Hubbard model the width of band of both spins is $4 t$, thus for $U<4 t$ it is possible to happen double particle occupation in a site having the system a lower energy than the state in single occupation on the top of the band. In the one-dimensional Falicov-Kimball model the width of the band of electrons is $4 t$, while the width of the band of ions is null. Thus, for any value of $U>0$, the double occupation of fermions leads to an energy of the system larger than that one that would occur in the case of simple occupation in the maximum level of energy of a ion because in the half-filled-band case, the maximum level of energy is zero. Also, crossing points clearly do not occur in the specific heat of the Falicov-Kimball model, differently from what happens with the Hubbard model, ${ }^{34}$ meaning that the ionic immobility impedes the occurrence of the condition $\partial C / \partial U=0$ at some definite temperature.

The inset in Fig. 2 shows details of the specific heat curve that we calculate for $U / t=4$ in low temperatures. It indicates that there is a great similarity with the form of the specific heat curve, i.e., a large broad maximum at low temperature, of the samarium hexaborite (SmB6) determined by Lawrence et al., ${ }^{35}$ and strengthens the evidences that the fermionic dynamics represented by the Falicov-Kimball model express an important part of the thermodynamic properties of mixed-valence materials.

\section{B. Internal energy}

Figure 3 shows the temperature dependence of the internal energy $(E)$ for the infinite chain. The sharp increase of 

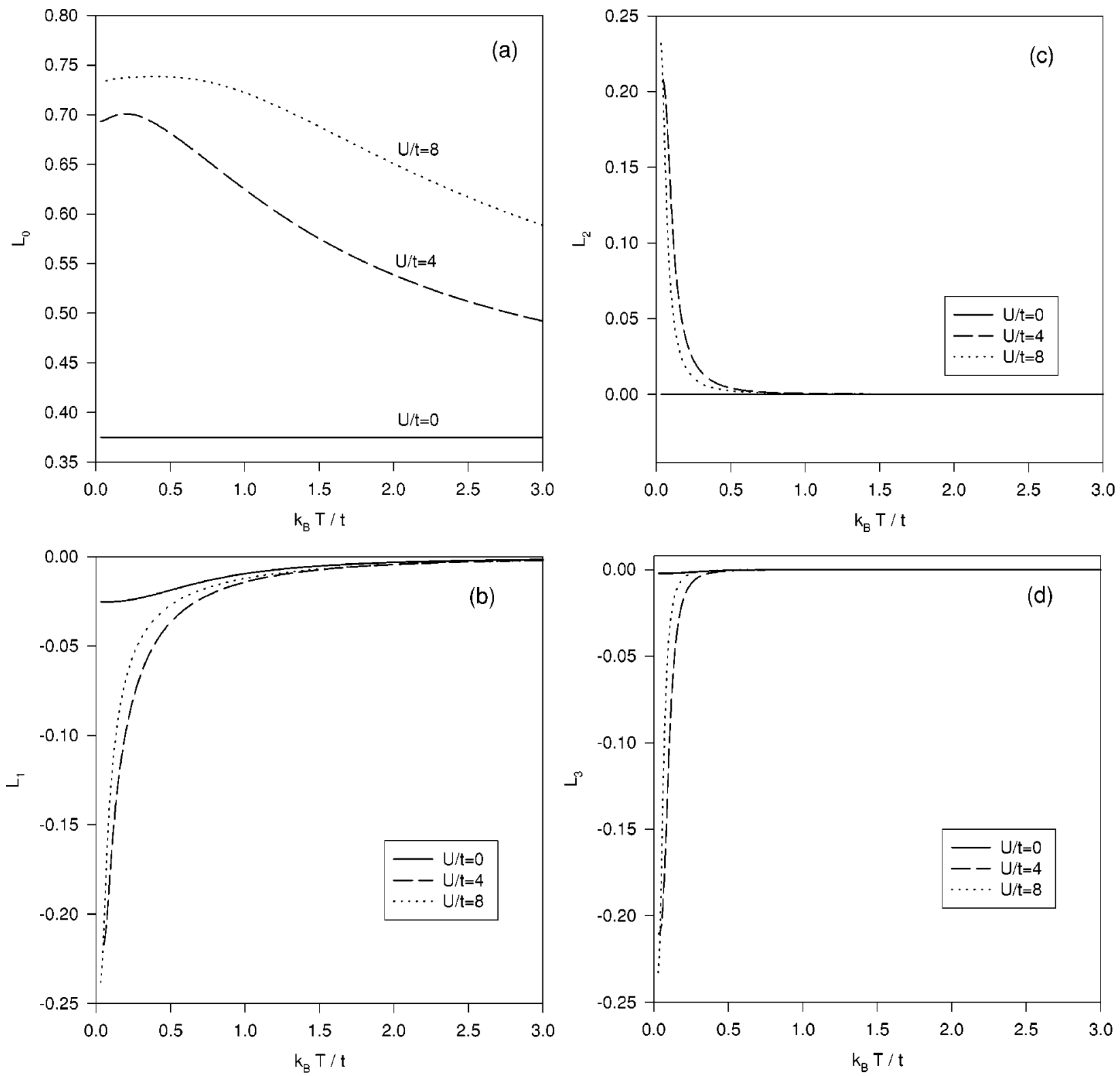

FIG. 6. Correlation functions $L_{\delta}$ of the infinite chain vs temperature for $U / t=0,4$, and 8. (a) $L_{0}$. (b) $L_{1}$. (c) $L_{2}$. (d) $L_{3}$.

the internal energy for $U / t>0$, which starts from $k_{B} T / t \gtrsim 0$, is related to the low-temperature peak in the specific heat, and it arises from electron-ion short-range ordering, while the sharp increase which starts from $k_{B} T / t \gtrsim 1$, is related to the high-temperature peak, and arises from single-particle excitations which form holes and double-occupied states (electron-ion). With the growth of $\mathrm{U}$, the effects of electronion short-range ordering and single-particle excitations are intensified, generating an increasing nonmonotonic behavior of the energy, a phenomenon that is consistent with the relative rise of the peaks and the decrease of the valley between the peaks of low and high temperature of the specific heat.

\section{Entropy}

Figure 4 shows the temperature dependence of the entropy for the same values of $U / t$ as before. Clearly, in the infinite chain, the entropy increases linearly at low temperature, and decreases with $U / t$. For $U / t>0$ two stages of excitations become distinct. The first stage up to $S / N k_{B}$ $\sim 0.69(\ln 2)$ is connected to the low-lying collective excitations and the second one from $S / N k_{B} \sim 0.69$ to $S / N k_{B}$ $\sim 1.39$ arises from charge transfer excitations, which create holes and doubly occupied states. The growth of $U$ provokes a nonmonotonic behavior of the entropy, in direct coherence with corresponding behavior of the internal energy and of the specific heat.

\section{Mean square fluctuation of the difference between the ion and electron numbers}

Denoting the difference between the ion and electron numbers operator by 


$$
\sigma=\sum_{i}\left(n_{f i}-n_{d i}\right)
$$

we calculate the mean square fluctuation of the difference between the ion and electron numbers using

$$
\chi=\frac{\mu_{B}^{2}}{k_{B} T}\left(\left\langle\sigma^{2}\right\rangle-\langle\sigma\rangle^{2}\right),
$$

where the symbol \langle\rangle denotes the average over the grand canonical ensemble.

Our calculations for $\chi$ are given in Fig. 5. The mean square fluctuation of the difference between the ion and electron numbers for the infinite chain diverges at $T=0$ for $U / t=0$, because a divergence exists in the band of the system for the half-filled-band case. But, under the effect of fermionic correlation it becomes finite at $T=0$ for $U / t>0$. Thus, the introduction of positive values of $U / t$ changes dramatically the fermionic structure, expressing the effect of the ionic immobility. In this case the difference with the results of the magnetic susceptibility for the infinite chain of the Hubbard model is significant. ${ }^{31}$ In the case of the Hubbard model the susceptibility always begins with a finite value at $T=0$.

The peak of $\chi$, and its nonmonotonic behavior with the growth of $U$, in the case of the Falicov-Kimball model for $U / t>0$, is consistent with the sharp increase of the internal energy at low temperature, and is a consequence of the collective excitations that lead to the disorganization of the alternate electron-ion short-range order. The magnetic susceptibility of mixed-valent SmB6 presents a broad maximum at low temperature ${ }^{35}$ that is quite similar to the peak presented for the curve $U / t=4$ of Fig. 5, and coherent with our previous comment for the case of the specific heat.

\section{E. Correlation functions}

Considering that the operator for the difference between the ion and electron numbers in the site $i$ is $\sigma_{i}=n_{f i}-n_{d i}$, we define the correlation function

$$
L_{\delta}(T)=\frac{3}{4 N} \sum_{i}\left\langle\sigma_{i} \sigma_{i+\delta}\right\rangle .
$$

The meaning of the quantity $L_{\delta}(T)$ is clear. First of all, $L_{0}(T)$ shows the magnitude of the difference between the ion and electron numbers at each site (or the degree of localization of fermions). For a completely localized fermion system in which each site is occupied by a single fermion, $L_{0}$ is equal to $3 / 4$, while for a non-interacting fermion system we have $L_{0}=3 / 8$. Therefore, the temperature dependence of $L_{0}$ gives information on the degree of localization of fermions. On the other hand, $L_{\delta}(T)(\delta \neq 0)$ is the correlation between the differences of the number of ions to the number of electrons at different sites as a function of temperature.

The temperature dependence of $L_{0}, L_{1}, L_{2}$, and $L_{3}$ for some typical values of $U / t$ is shown in Figs. 6(a)-6(d). From these figures we are able to draw the following conclusions.

(i) As the temperature is increased, $L_{0}$ for $U / t>0$ gradually decreases at high temperature, i.e., electrons gradually delocalize. Thus, we conclude that the high-temperature peak in the specific heat is associated with the gradual localization of electrons (or the gradual metal-insulator transition).

(ii) The temperature dependence with negative signs of $L_{1}$ and $L_{3}$, and positive sign of $L_{2}$, for $U / t>0$, all three with a significant magnitude in the region where the specific heat has the first peak, makes possible to construct a physical picture as follows: as the temperature goes down, each site is occupied by just one fermion, and at very low temperatures the alternate ion-electron short-range ordering becomes evident.

\section{CONCLUSION}

The thermodynamic properties of the one-dimensional half-filled-band Falicov-Kimball model has been studied using small-cluster exact-diagonalization calculations and the extrapolation to the infinite chain. The thermal properties of the specific heat, internal energy, entropy, mean square fluctuation of the difference between the ion and electron numbers, and correlation functions support a physical picture of a system that exhibits characteristic behavior of alternate electron-ion short-range ordering at low temperature, and gradual metal-isolator transition at high temperature. But, the model does not exhibit the crossing point of the specific heat curves, characteristic of the Hubbard model.

The results can be used to interpret much of the experimental data of compounds recently considered with the Falicov-Kimball model. For example, the specific-heat curve for samarium hexaborite $\left(\mathrm{Sm} B_{6}\right)$ presents a large broad maximum at low temperature similar to the curve shown in the inset of Fig. $2 .^{35}$

\section{ACKNOWLEDGMENTS}

We have benefited from discussions and collaborations with many colleagues. It is a pleasure to thank, especially, A. M. S. Macêdo and M. E. de Souza. This work was supported by the $\mathrm{CNPq}$ (Brazilian agency).

\footnotetext{
${ }^{1}$ L. M. Falicov and J. C. Kimball, Phys. Rev. Lett. 22, 997 (1969).

${ }^{2}$ M. Plischke, Phys. Rev. Lett. 28, 361 (1972).

${ }^{3}$ D. K. Ghosh, Solid State Commun. 18, 1377 (1976).

${ }^{4}$ A. C. Hewson and P. S. Riseborough, Solid State Commun. 22,
}

379 (1977).

${ }^{5}$ H. S. Wio, Z. Phys. B 50, 357 (1983).

${ }^{6}$ T. Kennedy and E. H. Lieb, Physica A 138, 320 (1986); E. H. Lieb, ibid. 140, 240 (1986). 
${ }^{7}$ U. Brand and R. Schimidt, Z. Phys. B 63, 45 (1986).

${ }^{8}$ U. Brand and C. Mielsch, Z. Phys. B 75, 365 (1989).

${ }^{9}$ J. K. Freericks and L. M. Falicov, Phys. Rev. B 41, 2163 (1990).

${ }^{10}$ P. de Vries, K. Michielsen, and H. de Raedt, Phys. Rev. Lett. 70, 2463 (1993).

${ }^{11}$ J. Lach, R. Lyzwa, and J. Jedrzejewski, Acta Phys. Pol. A 84, 327 (1993).

${ }^{12}$ R. Lyzwa and Z. Domański, Phys. Rev. B 50, 11381 (1994).

${ }^{13}$ P. Farkašovský, Phys. Rev. B 51, 1507 (1995).

${ }^{14}$ G. I. Watson and R. Lemanski, J. Phys.: Condens. Matter 7, 9521 (1995).

${ }^{15}$ J. K. Freericks, C. Gruber, and N. Macris, Phys. Rev. B 53, 16189 (1996).

${ }^{16}$ M. Park and J. Hong, J. Korean Phys. Soc. 33, 480 (1998).

${ }^{17}$ P. Farkašovský, Phys. Rev. B 57, 14722 (1998).

${ }^{18}$ M. S. Laad and M. van den Bossche, J. Phys.: Condens. Matter 12, 2209 (2000).

${ }^{19}$ N. Datta, A. Messager, and B. Nachtergaele, J. Stat. Phys. 99, 461 (2000).

${ }^{20}$ N. Datta, R. Fernández, and J. Fröhlich, J. Stat. Phys. 96, 545 (1999).

${ }^{21}$ P. Farkašovský, Phys. Rev. B 60, 10776 (1999).
${ }^{22}$ T. Portengen, T. Östreich, and L. J. Sham, Phys. Rev. Lett. 76, 3384 (1996).

${ }^{23}$ G. Czycholl, Phys. Rev. B 59, 2642 (1999).

${ }^{24}$ P. Farkašovský, Phys. Rev. B 59, 9707 (1999).

${ }^{25}$ K. Leung and F. S. Csajka, J. Chem. Phys. 108, 9050 (1998).

${ }^{26}$ C. Gruber and N. Macris, Helv. Phys. Acta 69, 850 (1996).

${ }^{27}$ W. Chung and J. K. Freericks, Phys. Rev. B 57, 11955 (1998).

${ }^{28}$ J. K. Freericks and V. Zlatić, Phys. Rev. B 59, 322 (1998).

${ }^{29}$ J. K. Freericks, C. Gruber, and N. Macris, Phys. Rev. B 60, 1617 (1999).

${ }^{30}$ P. Farkašovský, Phys. Rev. B 54, 7865 (1996).

${ }^{31}$ H. Shiba, Prog. Theor. Phys. 48, 2171 (1972); H. Shiba and P. A. Pincus, Phys. Rev. B 5, 1966 (1972).

${ }^{32}$ See also A. M. S. Macedo, M. C. dos Santos, M. D. CoutinhoFilho, and C. A. Macêdo, Phys. Rev. Lett. 74, 1851 (1995); A. M. C. de Souza, Phys. Rev. B 51, 1315 (1995).

${ }^{33}$ J. Hubbard, Proc. R. Soc. London, Ser. A 276, 238 (1963).

${ }^{34}$ N. Chanda, M. Kollar, and D. Vollhardt, Phys. Rev. B 59, 10541 (1999).

${ }^{35}$ J. M. Lawrence, P. S. Riseborough, and R. D. Parks, Rep. Prog. Phys. 44, 1 (1981). 